\title{
Compliance- und Integrity-Ansätze in der Unternehmensethik \\ Normenorientierung ohne Werte oder Werteorientierung ohne Nor- men?*
}

\section{LISA SCHÖTTL UND ROBERT RANISCH ${ }^{* *}$}

Compliance und Integrity in der Wirtschaftsethik gelten als zwei Ansätze einer verantwortlichen Unternehmenssteuerung. Compliance wird dabei häufig als eine regel- bzw. normenbasierte Strategie ausgewiesen, wohingegen Integrity als ein werteorientierter Ansatz verstanden wird. Gegenstand des Artikels ist die Frage, inwieweit aus einem Abgleich von Compliance- und Integrity-Strategien mit einer philosophischen Normen- und Werte-Unterscheidung Erkenntnisse für die Beziehung beider Unternehmensstrategien gewonnen werden können. Die konzeptionelle Analyse wird dabei Einsicht in ein sich ergänzendes Verhältnis erlauben und zugleich Grenzen beider Ansätze aufzeigen.

Schlagwörter: Integrity, Compliance, Werte, Normen, Unternehmensführung, Unternehmensverantwortung

\section{Approaches of Compliance and Integrity in Business Ethics: Norm-Orientation without Values or Value-Orientation without Norms?}

In business ethics Compliance and Integrity approaches are considered as two strategies of responsible organizational management. While Compliance has characteristics of a rule- or norm-based strategy, Integrity seems to resemble a value-based strategy. This paper discusses the questions to which extent the difference between Compliance and Integrity drawn along the lines of the philosophical distinction between norms and values can belp to clarify the relation of the respective accounts of business ethics. The conceptual analysis will shed light on their complementary nature and at the same time reveal some limits of both approaches.

Keywords: Integrity, Compliance, V alues, Norms, Management, Corporate Responsibility

\footnotetext{
* Beitrag eingereicht am 04.12.2015; nach doppelt verdecktem Gutachterverfahren überarbeitete Fassung angenommen am 09.05.2016.

** Lisa Schöttl, Konstanz Institut für Corporate Governance, Hochschule Konstanz, Brauneggerstr. 55, D-78462 Konstanz, Tel.: +49-(0)7531-206439, E-Mail: lisa.schoettl@htwg-konstanz.de, Forschungsschwerpunkte: Wirtschafts- und Unternehmensethik, insb. Corporate Governance, Corporate Responsibility und Integrity Management.

Robert Ranisch, Internationales Zentrum für Ethik in den Wissenschaften (IZEW), Universität Tübingen, Wilhelmstraße 19, D-72074 Tübingen, Tel.: +49-(0)7071-2975672, E-Mail: robert.ranisch@izew.uni-tuebingen.de, Forschungsschwerpunkte: Angewandte Ethik, insb. Ethik in Organisationen und Bioethik.
} 


\section{Einführung: Compliance und Integrity als Ansätze verantwortlicher Unternehmenssteuerung}

Eine gute Unternehmenssteuerung zeichnet sich unter anderem dadurch aus, dass sich ein Unternehmen an die gesetzliche Rahmenordnung hält und an Wertvorstellungen der Gesellschaft orientiert. In gegenwärtigen Debatten um die Verantwortung von Unternehmen werden diese Forderungen unter den Schlagwörtern Compliance und Integrity behandelt (vgl. u.a. Wieland/Steinmeyer/Grüninger 2014). Compliance (dt. Einhaltung) bezieht sich auf die Regeltreue eines Unternehmens, mit dem Ziel rechts- und regelwidriges Verhalten zu verhindern. Insbesondere durch diverse vergangene und gegenwärtige Skandale um die Verletzung fundamentaler Gesetze und Regeln durch Unternehmen hat das Thema Compliance in der Diskussion um die Verantwortung von wirtschaftlichen Akteuren an Prominenz gewonnen. Mittlerweile wird Compliance zu den wesentlichen Voraussetzungen guter Unternehmensführung gezählt, wie auch entsprechende Verweise im Deutschen Corporate Governance Kodex deutlich machen. ${ }^{1}$ Den Gegenstand von Compliance, also den Bereich, auf den sich die „Einhaltung“ bezieht, bilden hauptsächlich gesetzliche und regulatorische Vorgaben.

Mit Blick auf die Literatur wird mitunter ausgeführt, dass sich Compliance ausschließlich auf eine am geltenden Gesetz orientierte Verhaltensstrategie beziehe, die sich dann bestenfalls als eine an Verboten orientierte „Minimalmoral“ ausprägt (,if it's legal, it's ethi-

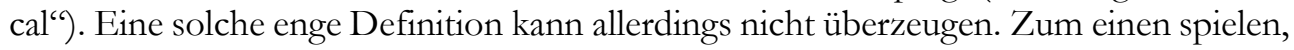
insbesondere im internationalen Aktionsbereich eines Unternehmens, immer mehr auch Regelungen des „Soft Law“ eine Rolle, also weiche Steuerungsmechanismen, die zwar nicht rechtsverbindlich sind, aber hinsichtlich der Gültigkeit gesetzesähnlichen Charakter haben. Dazu zählen diverse branchenweite und internationale Standards, wie z.B. die ILO Kernarbeitsnormen oder die UN Guiding Principles on Business and Human Rights, die insbesondere wegen ihrer breiten internationalen Anerkennung von Bedeutung sind. Zum anderen muss sich Compliance nicht nur auf das geschriebene Gesetz (,the letter of law“) beziehen, sondern wird sich mitunter auch nach dem Geist des Gesetzes (,the spirit of law“) richten (vgl. Steinmann/Olbrich 1998) und gesellschaftlichen Ansprüchen (Social Compliance) genügen müssen. Im Rahmen eines Compliance Managements werden schließlich auch außerhalb des Gesetzes liegende Normen beachtet, jedoch nur - und dies ist ein wesentliches Merkmal eines ComplianceAnsatzes -, insofern diese (zum Teil nur informellen) Regeln oder Erwartungshaltungen ein relevantes ökonomisches Risiko wie etwa einen Reputationsverlust für Unternehmen darstellen.

Kennzeichen eines Compliance-Ansatzes ist somit eine „Konformität mit extern auferlegten Standards“ (Paine 1994: 113; eigene Hervorhebung) unabhängig davon, ob diese Forderungen beispielsweise vom Gesetzgeber, von standardsetzenden Organisationen oder wesentlichen Stakeholdern des Unternehmens stammen. Im Fokus des Unterneh-

1 Im Deutschen Corporate Governance Kodex wird etwa der Vorstand einer Aktiengesellschaft explizit auf Compliance verpflichtet. Gemäß Ziffer 4.1.3 heißt es darin: „Der Vorstand hat für die Einhaltung der gesetzlichen Bestimmungen und der unternehmensinternen Richtlinien zu sorgen und wirkt auf deren Beachtung durch die Konzernunternehmen hin (Compliance).“ 
mens steht das Ansinnen, Regelverletzungen und mögliche damit verbundene Sanktionen zu vermeiden. Compliance stellt damit eine Grundvoraussetzung für eine erfolgreiche Unternehmensführung dar, denn Compliance-Verstöße können für ein Unternehmen folgenschwer oder gar existenzbedrohend sein, da sie nicht selten sowohl strafrechtliche (Haftstrafen etc.) als auch ökonomische (Geldbußen, Umsatzeinbrüche etc.) Folgen nach sich ziehen und die Reputation für lange Zeit beschädigen können.

Mit der wachsenden Aufmerksamkeit für das Thema (Non-)Compliance ist auch die Frage in den Mittelpunkt gerückt, ob es nicht nur zur Verantwortung eines Unternehmens gehört, rechtliche und gesellschaftliche Anforderungen in seinem Handeln zu berücksichtigen, insofern sich diese ökonomisch lohnen, sondern sich auch an moralischen Werten zu orientieren. Denn, so die häufig geäußerte Meinung, es gehe letztlich nicht nur darum, dass sich ein Unternehmen an Regeln hält, sondern dass es sich bewusst an Werten ausrichtet, wie sie etwa auch Gesetzen und regulatorischen Anforderungen zugrunde liegen (vgl. exemplarisch Wieland 2008; Grüninger 2014). Hier hat der Begriff der Integrität an Bedeutung gewonnen und soll zum Ausdruck bringen, dass ein Ziel der Bemühungen eines Unternehmens in diesem Bereich nicht die bloße Regeleinhaltung - die Vermeidung von Non-Compliance - sein sollte; es wird vielmehr eine spezifische werteorientierte Haltung des Unternehmens gefordert.

Die Unterscheidung zwischen einem Compliance- und einem Integrity-Ansatz hat Lynne Sharp Paine in ihrem vielbeachteten Artikel Managing for Organizational Integrity (1994) bekannt gemacht. Dort verdeutlicht sie, dass eine Compliance-Strategie nur eine mögliche Herangehensweise an Fragen einer verantwortlichen Unternehmensführung darstellt und plädiert stattdessen für eine Integrity-Strategie, bei der ein Unternehmen sein Handeln an selbst gesetzten Standards und Unternehmenswerten ausrichtet und damit moralisch verantwortliches Handeln im und durch das Unternehmen ermöglicht (vgl. Paine 1994: 113). ${ }^{2}$ Integrity-Strategien bezeichneten eine weit über das legale Recht hinausgehende Werteorientierung, die das je eigene Streben der unternehmerischen Akteure zum Ausdruck bringe. Im Unterschied zu Compliance, wo Regeln im Zentrum stehen, nehmen bei Integrity nach Paine Werte die zentrale Position ein.

Darüber hinaus wird die Differenz zwischen Compliance und Integrity in der Literatur entlang einer Vielzahl an zum Teil komplementären Begriffspaaren gezeichnet: konformes Verhalten vs. internalisiertes Handeln; extrinsische Motivation vs. intrinsische Motivation (vgl. Steinmann/Olbrich 1998: 77); Sanktionen vs. Anreize; reaktiv vs. proaktiv (vgl. Rasche/Esser 2007: 108-109); hypothetische Zwecke vs. kategorische Werte (vgl. Beschorner 2005: 47); strictly defined vs. dynamic (vgl. Rasche/Esser 2007: 112). Damit sind zugleich verschiedene Dimensionen beider Ansätze angesprochen. Nicht eindeutig geklärt ist allerdings das Verhältnis von Compliance zu Integrity, da beide Orientierungen zwar einerseits durch Zielsetzung und Mittel voneinander unterschieden werden können, andererseits jedoch Compliance meistens auch für ein Streben nach Integrität

2 Obgleich Paine betont ,integrity is based on the concept of self-governance“, scheint es ihr nicht darum zu gehen, dass Integrity-Strategien auf beliebigen selbst gesetzten Werten aufbauen, sondern auf solchen Werten, die ein „ethically sound behavior“ fördern (1994: 111). Offen bleibt allerdings, wie sichergestellt werden kann, dass diese selbstgesetzten Werte stets moralischen Anforderungen genügen. 
vorausgesetzt wird. ${ }^{3}$ Mit Ausnahme weniger Autoren wie Steinmann und Olbrich (1998: 69), die meinen, ,the two types of ethical management are (analytically) mutually exclusive", wird in aller Regel angenommen, dass sich beide Strategien zumindest teilweise überlappen oder ergänzen können. Thielemann geht dabei davon aus, dass es sich keineswegs um ,unterschiedliche Ansätze“ handelt, sondern sich Compliance und Integrity „wechselseitig bedingen“ (Thielemann 2005: 31f.; vgl. Beschorner 2005) und auch Rasche und Esser betonen ein Ergänzungsverhältnis (vgl. Rasche/Esser 2007: 115). Neben diesen systematischen Annäherungen an die Beziehung von Compliance und Integrity zeigt sich zudem eine normative Verhältnisbestimmung, die bereits Paine äuBerte: ,an integrity strategy is broader, deeper, and more demanding than a legal compliance initiative" (Paine 1994: 111). Integrity wäre demnach eine Compliance-Strategie mit einem Plus; Integrity müsse Compliance erweitern und verbessern (vgl. Grüninger 2011: 10).

Diese gegenseitige Ergänzung von Compliance und Integrity, die im Zuge der wissenschaftlichen Debatte gezeichnet wird, ist vielschichtig. Wie die vorigen Ausführungen bereits andeuteten, können diverse Dimensionen ausgemacht werden, die das Verhältnis beider Ansätze charakterisieren und deren Abhängigkeit aufzeigen (vgl. u.a. Thielemann 2005; Rasche/Esser 2007; Wieland 2014). In philosophischer Hinsicht interessant ist dabei die oft wiederkehrende und mit ethischen Kategorien vorgenommene Differenzierung der zwei Ansätze: So scheint eine Compliance-Orientierung in der Unternehmenssteuerung Merkmale einer regel- bzw. normenorientierten Strategie aufzuweisen, wohingegen Integrity an eine werteorientierte Strategie erinnert. Damit ist ein wesentlicher Anknüpfungspunkt der Debatte um Compliance und Integrity an die philosophische Diskussion um Werte und Normen gegeben, der womöglich weitere Einsichten für das Verhältnis der beiden Unternehmensstrategien bereithalten kann. In dieser Hinsicht verfolgt der Beitrag ein zweifaches Anliegen. Zunächst soll in explanatorischer Absicht die Differenz von Compliance und Integrity mit der Unterscheidung von Normen und Werten abgeglichen werden. Dabei wird sich zeigen, dass sich aus der Normen-WerteKonstellation Einsichten für das Ergänzungsverhältnis von Compliance und Integrity ableiten lassen, welche in einem zweiten Schritt ausgeführt werden.

\section{Normen und Werte als Differenzkriterien von Compliance- und Integrity-Ansätzen?}

Um Einsichten für das Ergänzungsverhältnis von Compliance und Integrity zu gewinnen, soll an dieser Stelle zunächst der Versuch unternommen werden, beide Ansätze mit Kategorien von Normen und Werten abzugleichen, wie sie insbesondere in der (Moral-)Philosophie, aber auch der Soziologie Verwendung finden. Dieser Abriss kann dabei nur kursorisch erfolgen. Sowohl Normen- als auch Werturteile beziehen sich schließlich auf vielfältige Gegenstandsbereiche. Neben den hier im Zentrum stehenden Fragen der Ethik und Moral, können sich Normen und Werte etwa auch auf ökonomische, technische, prudentielle oder ästhetische Bereiche beziehen. Zudem zeigt sich,

3 So auch von Paine, die zum einen für einen Wechsel von einer Compliance- zu einer IntegrityStrategie plädiert, zum anderen aber gleichzeitig Compliance als notwendigen Bestandteil von Integrity ansieht (Paine 1994: 113; vgl. hierzu auch Thielemann 2005: 36f.). 
dass - obgleich wir es hier mit zwei Grundbegriffen der praktischen Philosophie zu tun haben - selten die Frage nach der Unterscheidung von Normen und Werten explizit adressiert wird. ${ }^{4}$

Folgen wir dem Sozialphilosophen Hans Joas, der sich intensiv mit dem Verhältnis zwischen Normen und Werten auseinandergesetzt hat, erinnert insbesondere der von ihm gezeichnete Unterschied zwischen Normen als dem Restriktiv-Obligatorischen und Werten als dem Attraktiv-Motivierenden an die aus der Literatur bekannten Kennzeichen von Compliance- und Integrity-Ansätzen (vgl. Joas 1997: 288). Compliance, so wurde betont, stellt schließlich ein gebotenes Moment in der Unternehmenssteuerung dar, welches ein Unternehmen restringiert und eine „Schließung der Organisation für ethisch verfehlte Handlungsoptionen“ (Thielemann 2005: 37; vgl. Rasche/Esser 2007: 114; Ulrich 2004b: 31) bewirkt. Eine Orientierung an Integrity bezieht sich dagegen auf die kollektive, identitätsstiftende Entwicklung spezifischer Unternehmenswerte, die eine „Öffnung der Organisation für ethische Einsichten“ (Thielemann 2005: 37) fördert. Dementsprechend führt Paine (1994: 111) aus: „From the perspective of integrity, the task of ethics management is to define and give life to an organization's guiding values". In ganz ähnlicher Weise sehen auch Rasche und Esser die Verbindung von Normorientierung und Compliance, denn dieser Ansatz „focuses on prescriptions“ und ,assumes third party control“. Integrity wiederum „depends on enacted values, and not on strictly defined external norms" (Rasche/Esser 2007: 111f.). Compliance stellt sich demnach als Regelbefolgungsansatz dar, Integrity dagegen als wertegetriebener Ansatz (vgl. Beschorner 2005: 48; Wieland 2014: 32). Letztere Herangehensweise ist dabei dadurch gekennzeichnet, dass Unternehmen nicht nur gesetzlich und moralisch konform handeln, sondern ein Bewusstsein für das Richtige ausprägen, und dies nicht allein als strategisches Mittel zur Erlangung bestimmter (ökonomisch profitabler) Zwecke betrachten (vgl. Beschorner 2005: 47). Die verinnerlichten Unternehmenswerte bringen so zum Ausdruck, wofür ein Unternehmen steht: „what a company is and what it stands for" (Paine 1994: 111). Das Handeln stimmt hier nicht nur mit dem moralisch Richtigen überein, sondern zeugt auch von einer spezifischen Orientierung am Guten, d.h. dass eine Haltung des aufrichtigen Strebens nach Werten Teil des Selbstverständnisses ist.

Um Normen und Werte als Differenzkriterien von Compliance- und Integrity-Ansätzen noch klarer zu zeichnen, sollen mit Blick auf die moralphilosophische Literatur sowie die moralische Praxis weitere Charakteristika rekonstruiert werden (vgl. Fassio 2013, 150ff.). Schauen wir zunächst auf die alltägliche Verwendung, so fällt auf, dass Normen (Regeln, Vorschriften usf.) mit deontischen Begriffen ausgedrückt werden, nach denen etwas geboten, verboten oder erlaubt ist. Werte dagegen werden mit evaluativen Begriffen beschrieben, nach denen etwas gut, schlecht bzw. böse oder neutral ist. Während deontische Begriffe einen Handlungsbezug aufweisen - sie stehen mit dem Tun und Unterlassen von Akteuren in Verbindung und geben diesbezüglich verbindliche Anweisungen -, ist der Anwendungsbereich von evaluativen Begriffen vielfältiger. Nahezu

4 Für eine philosophische Übersicht zum Verhältnis von Normen und Werten, siehe Horster (2007) sowie für Werte in der Ethik Höffe (2002) und Krijnen (2002). Zur Unterscheidung von Normen- und Werturteilen bzw. deontischen und evaluativen Begriffen, Tappolet (2013), Fassio (2013); im Kontext der Unternehmensethik siehe etwa Talaulicar (2006: 345-350). 
jedes Objekt und jeder Zustand kann Gegenstand der Bewertung sein. Im Zusammenhang mit moralischen und moralrelevanten Wertausaussagen können wir etwa von guten oder schlechten Handlungen, Zielen, Absichten, Einstellungen, Zuständen usf. sprechen. Zudem ist das Vokabular der Wertbegriffe reicher als dasjenige der deontischen Konzepte: etwas oder jemand kann nicht nur als gut oder schlecht, sondern auch als verlässlich, aufrichtig, effizient usw. bewertet werden. Werte manifestieren dabei stets einen erstrebens- oder wünschenswerten Zustand und drücken aus, dass etwas der Fall sein soll. Von daher wird über sie auch als „Orientierungsdirektive“ (Krijnen 2002: 528) oder „Leitvorstellungen“ (Höffe 2002: 290) gesprochen. Im Vergleich zu Normen sind sie dabei aber nicht unmittelbar handlungsverbindlich. Werte können vielmehr auch den Charakter von Idealen annehmen, wohingegen Normen meist handfeste Forderungen darstellen. Charakteristischerweise werden diese in Form von Vorschriften oder Gesetzen formuliert und bringen zum Ausdruck, dass etwas getan werden soll.

Ein weiteres typisches Unterscheidungsmerkmal zeigt sich in der Kodierung von Normen und Werten (vgl. Habermas 1992: 311). Werte können mehr oder weniger realisiert werden und eine bestimmte Strategie als besser oder schlechter als ihre Alternativen bewertet werden. Demgegenüber sprechen wir hinsichtlich Normen nicht davon, dass eine Handlung mehr oder weniger geboten ist. ${ }^{5}$ Das deontische Vokabular ist binär, Handlungen gelten als verboten oder eben nicht. Normen sind dabei stets präskriptiv und stehen in Verbindung mit der Idee der Pflicht. Werte sind evaluativ und durch ein Streben nach dem Guten ausgezeichnet bzw. nach etwas, was als gut erachtet wird. ${ }^{6}$ Deutlich wird dabei auch, dass Werte und Werturteile häufig mit spezifischen Haltungen und affektiven Zuständen in Verbindung stehen (vgl. Tappolet 2015). Wenn ich etwas als gut oder wertvoll erachte, bin ich in der Regel in einem bestimmten emotionalen Zustand hinsichtlich des Bewertungsgegenstandes. ${ }^{7}$ Als geteilte oder kollektive Werte, wie etwa in Unternehmen, sind diese häufig Quelle der Identitätsbildung und gemeinschaftlicher Sinnstiftung.

\section{Annäherung an das Verhältnis von Normen und Werten in der (Diskurs-) Ethik}

Ausgehend von der Unterscheidung von Normen und Werten gilt es nun sich einer Verhältnisbestimmung zuzuwenden. Wenn zumindest über einige der Merkmale beider Begriffe Konsens besteht, herrscht in der Philosophie über deren Verhältnis Uneinigkeit (vgl. Horster 2007). Insbesondere ob Normen in der Ethik der Vorrang vor Werten

5 Dies bedeutet nicht, dass wir nicht legitimer Weise von einer Hierarchie im Fall von Normenkonflikten ausgehen können. So kann es sein, dass eine Norm („Du sollst nicht töten.“) mehr Gewicht hat als eine andere Norm („,Du sollst nicht lügen.“). Dies bedeutet allerdings nicht, dass eine der konfligierenden Normen damit „,weniger“ richtig und geboten ist, sondern dass diese unter bestimmten Bedingungen untergeordnet wird (vgl. Tappolet 2013).

6 Zu den umstrittensten Themen der Ethik gehört dabei freilich die Frage der Realität von Werten: werden diese „erfunden“ oder in der Welt „entdeckt“?

7 An dieser Stelle muss freilich eine deskriptive Perspektive auf Werte unterschieden werden. So kann ich etwa beschreibend wiedergeben, dass im Unternehmen dieser oder jener Faktor (Effizienz, Zuverlässigkeit etc.) ein hohes Gut darstellt, ohne dass eine solche Evaluation zum Set der von mir geteilten Werte gehört. 
gebührt oder vice versa, ist weiterhin eine offene Grundsatzdebatte, die an dieser Stelle freilich nicht entschieden werden kann. Allerdings können Einsichten über gemeinhin als gescheitert geltende Verhältnisbestimmungen für die Frage nach der Rolle einer werteorientierten Unternehmensethik nutzbar gemacht werden. Im folgenden Abschnitt wird dazu eine Konzeption herangezogen, wie sie im Rahmen der Diskursethik entwickelt wurde. Diese Fokussierung hat zwei Gründe. Zum einen stellt die Diskursethik eine der wirkmächtigsten Moraltheorien dar, die insbesondere durch die Vermittlung von Peter Ulrich und anderen auch in die Wirtschaftsethik Eingang gefunden hat (vgl. Ulrich 2008; König 2001). Zugleich hat sich Jürgen Habermas als Hauptvertreter ${ }^{8}$ der Diskursethik an verschiedenen Stellen klar zum Verhältnis von Normen und Werten in der Ethik positioniert (vgl. Habermas 1991, insb. 100-118; 1992; 2000). Die von ihm vorgeschlagene scharfe Trennung beider Konzepte sowie die Marginalisierung von Werten in der Ethik wird als (negative) Blaupause dienen, um anschließend die Unterscheidung von Compliance und Integrity zu verhandeln.

In Faktizität und Geltung (1992) spezifiziert Habermas einige uns zum Teil schon vertraute Unterscheidungsmerkmale:

„Normen und Werte unterscheiden sich (...) erstens durch ihre Bezüge zu obligatorischem bzw. teleologischem Handeln; zweitens durch die binäre bzw. graduelle Kodierung ihres Geltungsanspruchs; drittens durch ihre absolute bzw. relative Verbindlichkeit und viertens durch die Kriterien, denen der $\mathrm{Zu}-$ sammenhang von Norm- bzw. Wertsystemen genügen muß.“ (Habermas 1992: 311)

Wesentliche Unterscheidungsmerkmale zeigten sich demnach darin, dass Normen verbindlich das Gebotene oder Verbotene ausdrücken, was universelle Geltung beansprucht. Werte dagegen stehen stärker mit Handlungszielen und mit als erstrebenswert angesehenen Gütern in Verbindung, deren Realisierung gegebenenfalls auch nur annäherungsweise möglich oder praktikabel ist. Im Rahmen der Diskursethik kommt Habermas dabei zu dem Schluss, dass der Primat Normen (,dem Gerechten“) und nicht Werten („dem Guten“) gebührt. ${ }^{9}$ Nur Normen könnten die für die Moral notwendige objektive Geltung und Universalität beanspruchen und „die Zustimmung aller Betroffenen als Teilnehmer eines praktischen Diskurses finden" (Habermas 1996: 49). Nur Normen, d.h. auch Regeln oder Prinzipien, verpflichten kategorisch und hätten eine unbedingte Sollgeltung. Während sich Werte nach Habermas durch ihre Partikularität auszeichnen - sie sind nicht notwendig gut für alle, sondern gut für einige - und im Zusammenhang mit Fragen von Identität, Lebensform und dem individuell oder kollektiven

Neben Habermas hat insbesondere Karl-Otto Apel zur Entwicklung der Diskursethik beigetragen. Auch wenn bei Habermas die Programmatik der Diskursethik wohl in den Hintergrund gerückt ist, kann sie dennoch als der zentrale Beitrag der deutschsprachigen Philosophie zur Ethik des letzten Jahrhunderts verstanden werden und beeinflusst auch die angewandte Ethik bis heute. Für eine Übersicht zu Entwicklungen und verschiedenen Ausarbeitungen der Diskursethik siehe Gottschalk-Mazouz (2000).

9 Habermas identifiziert dabei Fragen des Gerechten mit „Moral“ und Fragen des Guten mit „Ethik“ (Habermas 1991: 100-118). Wenn nicht anders gekennzeichnet, werden wir von dieser Unterscheidung absehen und Ethik als Reflexion auf Moral verstehen. 
Guten stehen, bestimmen Normen die allgemeinverbindlichen Regeln des Zusammenlebens, unabhängig von konfligierenden Einzelinteressen. So kann es als Anliegen der Diskursethik verstanden werden, gerade ein formales Verfahren zu formulieren, um derartige allgemeinverbindliche Gebote von „bloßen“ Werten sowie jeder Form von beliebigen Präferenzen zu unterscheiden. Im Rahmen dieser Prozedur würden schließlich „partikulare Wertgesichtspunkte als nicht konsensfähig am Ende herausfallen“ (Habermas 1983: 113).

So wirkmächtig die von Habermas getroffene Unterscheidung von Normen und Werten und dem damit einhergehenden Postulat eines Primats des Gerechten vor dem Guten ist, so sehr ist die Verhältnisbestimmung Gegenstand andauernder Kritik (vgl. exemplarisch Putnam 2002; Joas 1997). ${ }^{10}$ Während für den Fortgang der Untersuchung eine tiefergehende Analyse der Problematik der Habermas'schen Verhältnisbestimmung nicht notwendig ist, sollen zwei Gesichtspunkte der Kritik seiner Diskursethik hervorgehoben werden und als erkenntnisleitend für die Auseinandersetzung mit der Unterscheidung von Compliance und Integrity dienen. Diese ergeben sich aus der für die Diskursethik charakteristischen Architektur, die eine Berührung von Normen und Werten unterbindet und letzteren schließlich den Weg in die Ethik versperrt. Insbesondere gegen eine solche Marginalisierung des Guten, d.h. von Werten in der Ethik, hat sich folglich auch starker Widerstand gezeigt. Dieser kulminiert in dem im Folgenden geteilten Postulat eines Ergänzungsverhältnisses zwischen dem Gerechten und dem Guten oder, anderes formuliert, in der Einsicht, dass in der Ethik „Werte und Normen vorkommen" müssen (Joas 1997: 287).

Für den hiesigen Zweck der Argumentation sollten dabei zwei systematisch zusammenhängende Gesichtspunkte der Kritik separat verhandelt werden: Die Ausgrenzung von Werten aus Normen führt erstens zu einem Motivationsproblem für moralisches Handeln, das sowohl Individuen als auch Kollektive betrifft, und zweitens zu einem Formalismusproblem bezüglich der Gehalte von (moralischen) Normen.

Versteht man Moral wie Habermas als ein System von allgemeingültigen, universalistischen Geboten und Verboten, deren Geltung in einer formalen Prozedur des Diskurses unter wohldefinierten Bedingungen geprüft wird, stellt sich die Frage, wie entsprechend gültige Normen Akteure überhaupt motivieren sollen, moralisch zu handeln (vgl. ReeseSchäfer 1997: 134-143). Wie Habermas bezüglich seiner Konzeption selbst erkennt, weist die Orientierung an Normen nur „die schwache motivierende Kraft guter Gründe“ auf (Habermas 1992: 183). Ohne eine spezifische geteilte Wertehaltung, welche die moralische Perspektive zu einem Teil der Identität werden lassen könnte, bleibt allerdings undurchsichtig, wie Normen nicht bloß instrumentell, sondern aus sich heraus Handeln motivieren können und sich eine Orientierung am Guten einstellen soll. Fraglich ist damit, auf welcher Grundlage Subjekte überhaupt einer Norm zustimmen sollten, insofern diese nur geringfügig mit ihren je eigenen Werten, respektive mit ihren Konzepten des Guten in Verbindung stünde (vgl. Lumer 1997). Peter Ulrich ist dabei

10 Zudem ist selbst Habermas in seinen jüngeren Schriften von der scharfen Gegenüberstellung von dem Gerechten und dem Guten abgerückt und hat nunmehr den Bereich von universellen Normen, also für ihn die „Moral“, in eine spezifische werteorientierte Lebensführung eingebettet (vgl. Habermas 2001). 
zuzustimmen, wenn er hinsichtlich der Diskursethik anmerkt, dass es „für freie Subjekte kein absolutes (,zwingendes') Müssen“ gibt, sondern ,allenfalls (nur) ein vernünftiges Wollen“. Nur für ein „transzendentales Subjekt“" im kantischen Sinne, „wären ,reine“ Vernunftgründe schon hinreichende, ja ,zwingende" Motive“. Die in der Diskursethik enthaltene Vorstellung einer „transzendentalen Nötigung“ gelingt nach Ulrich dagegen „gerade in einer modernen Welt nicht.“ Eine moralische Haltung könne folglich ,,nicht oder zumindest nicht primär aus absoluten rationalen Verbindlichkeitsansprüchen" motiviert werden, sondern nur aus Erfahrungen, die „,identitätsprägend sind (...) und unsere Vorstellung von einem guten, sinnvollen und erfolgreichen Leben bestimmen" (Ulrich 2004a: 25f.). Motive für moralisches Handeln bedürfen demnach einer Einbettung in bestimmte Werteorientierungen und -haltungen.

Zugleich, und dies scheint in gewisser Weise die inhaltliche Kehrseite des Motivationsproblems zu sein, scheint ein normenorientierter Ansatz ohne eine Komplementierung durch Werte inhaltlich unbestimmt. Joas betont hierbei zu Recht, dass das ,universelle ,Normenraster' (der Diskursethik) gar nichts zu prüfen hätte, wenn der Handelnde nicht auf verschiedene Vorstellungen vom Guten“, d.h. auf Werte, „hin orientiert wäre, bei denen er nicht sicher sein kann, ob sie unter dem Gesichtspunkt des Rechten akzeptabel sind“" (Joas 1997: 269). Eine Ethik ohne Werte scheint sich auf ein System von Prozeduren zur Prüfung von rein formalen Normen zu beschränken. Bereits aus systematischer Sicht scheinen Normen vielmehr auf spezifische Werte zu verweisen und deren Realisierungsbedingungen zu konkretisieren. Normen enthalten stets evaluative Anteile, ohne die sie gar nicht in einem materiellen Sinne gefüllt werden könnten (vgl. Putnam 2002: 118f.).

Wir können nun aus der philosophischen Auseinandersetzung mit dem Werte- und Normenverhältnis Einsichten für die Rolle von Werten im Rahmen der Auseinandersetzung mit einer Unternehmenssteuerung erlangen. Die beiden oben genannten Punkte sollen im Folgenden erläutert und insbesondere in ihrer Bedeutung für das Verhältnis von Compliance- und Integrity-Ansätzen untersucht werden.

\section{Bedeutung der philosophischen Analyse für Compliance- und Integrity-Ansätze}

\subsection{Zur motivationalen Funktion von Werten in der Unternehmens- steuerung}

Wenn ein Unternehmen anstrebt, regelkonformes und verantwortliches Handeln zu fördern, dann ist zur Realisierung dieses Vorhabens die Motivation der Unternehmensmitglieder zentral. Die ausschließliche Orientierung an bestimmten extern vorgegebenen Standards oder positivem Recht, wie es in Unternehmen mit einer ComplianceOrientierung üblich ist, kann die Unternehmensmitglieder nur schwerlich aus sich heraus bewegen, entsprechend zu handeln. Deshalb bedürfen derartige legalistische Ansätze häufig der Androhung von Sanktionen, um das gewünschte Verhalten zu erzielen. Bei einer solchen Incentivierung eines regelkonformen Verhaltens kann jedoch nicht mehr in einem anspruchsvollen Sinne von der Wahrnehmung von Verantwortung gesprochen werden, da sich hier, wenn überhaupt, ein rein instrumenteller Bezug zur Mo- 
ral manifestiert. Dieser Einsicht unterliegt die (kantische) Überzeugung, dass von moralischer Qualität nur ein nicht-opportunistisches Verhältnis zur Moral sein kann. ${ }^{11}$ Incentivierungsstrategien können zwar als Instrumente auch für eine moralisch verantwortliche Unternehmenssteuerung hilfreich sein, aber nur wenn sie dabei zur Prägung und Festigung einer wertebasierten Unternehmenskultur dienen. ${ }^{12}$

Um das gewünschte Handeln nicht bloß durch entsprechende Sanktionsandrohungen zu lenken, sondern wirksam - d.h. auch aus Überzeugung - zu erreichen, ist die Einbeziehung von Werten erforderlich, die zur Identitätsstiftung beitragen und den Sinn der Normeinhaltung zur Geltung bringen (vgl. Grüninger 2014: 63; Wieland 2014: 20). Nur wenn ein Akteur versteht, warum eine Norm eingehalten werden soll und entsprechende Gründe vor dem Hintergrund seiner Werteorientierung annehmen kann, wird er die nötige Motivation aufbringen, diese wirksam werden zu lassen, wie sich auch aus der Kritik an der Konzeption von Werten und Normen durch Habermas ergibt. Dazu ist eine Vorstellung des Guten, von Idealen notwendig, die in Werten zum Ausdruck kommen.

Des Weiteren ist eine Werteorientierung auch deshalb für Organisationen relevant, weil nur mit einer entsprechenden moralisch fundierten Haltung des Unternehmens die angestrebte Zuverlässigkeit im Handeln der Unternehmensmitglieder erzeugt werden kann. Wenn eine Organisation ihr Streben zwar an gesellschaftlich oder moralisch wünschenswerten Zwecken orientiert, diese aber nur als Mittel zur Reputationssteigerung oder zur Vermeidung von Strafen versteht, scheint dieses Handeln kaum zuverlässig, insofern die Konformität mit dem moralisch Richtigen stets vom Umstand der versprochenen Profite abhängig ist. Vielmehr muss eine Wertbindung, qua Unternehmenskultur, als eine verlässliche Strategie auf das Handeln des Unternehmens wirken und dabei zumindest zum Teil von moralischen Wertehaltungen geprägt sein (vgl. u.a. Grüninger 2014), die, wie skizziert, etwas evaluativ Gutes ausdrücken. Diese Überlegungen machen deutlich, dass erst ein Integrity-Ansatz, der die Einbindung von moralisch fundierten Werten in die Unternehmenssteuerung ernst meint, die Erfordernisse einer wirklich pflicht- und verantwortungsbewussten Unternehmensführung einlösen kann.

Diese Erkenntnis lässt sich auch mit der Bedeutung der Rede von „Integrität“ verdeutlichen. Entsprechend gängiger Definitionen von Integrität meint der Begriff (lat. ,integritas" = Ganzheit, Einheit) meist die Orientierung einer Person an von ihr geteilten Werten zusammen mit einer konsequenten Ausrichtung an diesen Werten, auch und

Für Compliance-Ansätze, wie Paine deutlich herausstellt, wird häufig das Motiv ,avoiding legal sanctions" (Paine 1994: 111) als zentral herausgestellt. Bei dieser Strategie kann es zwar sein, dass Unternehmen scheinbar moralisch richtig agieren, genau genommen handelt es sich hierbei aber gar nicht um eine unternehmensethische Strategie. Diese Erkenntnis wird auch von Thielemann (2005: 32) geteilt, wenn er im Hinblick auf einige Compliance-Strategien rhetorisch fragt, inwieweit „,man denn hier überhaupt von einem ,Ethikansatz' sprechen kann“.

12 Damit zeigt sich auch bei Integrity-Ansätzen eine Spannung, insofern diese „die Integration der ethischen Gesichtspunkte in die Unternehmensorganisation vermittels Anreizen bzw. Belohnung“ (Thielemann 2005: 42) bemühen. Wenn die Motivation der Unternehmensmitglieder zur Anerkennung bestimmter Werte einzig die Hoffnung auf Belohnung ist, wäre eine solche Ausrichtung vielmehr ein moralisches Lippenbekenntnis. Dies bedeutet freilich nicht, dass entsprechende Anreizsysteme nicht wirksam sein oder mittelfristig auch einen Beitrag zur Prägung einer moralischen Unternehmenskultur leisten können. 
gerade im Angesicht von Widerständen (vgl. u.a. McFall 1987: 8f.; Halfon 1989: 46f.; Cox et al. 2003: 7f.). Eben genau dann, wenn bestimmte beispielsweise ökonomische Faktoren oder „Sachzwänge“ gegen das moralisch Gute drängen und dieses dennoch bevorzugt berücksichtigt wird, zeigt sich die moralische Integrität einer Organisation, respektive ihrer Mitglieder.

Einschränkend muss allerdings hinterfragt werden, inwieweit ein werteorientierter Ansatz und die damit angesprochene Motivation der Unternehmensmitglieder alleine die Verbindlichkeit garantieren kann, die an eine moralische sowie auch ökonomisch erfolgreiche Unternehmessteuerung gestellt wird. Die im Zusammenhang mit Habermas formulierten Charakteristika von Werten machen gerade deutlich, warum es etwa schwierig wäre von einer kategorischen Werteorientierung zu sprechen..$^{13}$ Es ist Kennzeichen von Werten bzw. von Wertesystemen, dass diese plural sind und nicht notwendig einen Anspruch auf Konsistenz erheben (vgl. Cox et al. 2003: 19f.; Gottschalk-Mazouz 2000: 255f.). Für die Ausrichtung eines Akteurs wie etwa ein Unternehmen ist es legitim, dass sich dieser an einer Vielzahl an (moralischen, ökonomischen etc.) Werten ausrichtet, die seine Identität und sein Handeln auf authentische Weise auszeichnen (vgl. Cox et al. 2003: 8). Gerade im Falle von möglichen Wertekonflikten, etwa zwischen vom Unternehmen angestrebten ökonomischen und sozialen Werten, ist aus Sicht eines wertezentrierten Ansatzes keine Vorrangregel zu entnehmen, nach der stets diese oder jene Werte den Vorzug verdienen müssten, und ebenso ist nicht eindeutig, welche Werte letztlich den motivationalen Ausschlag geben. Vielmehr ist es für ein Wertesystem markant und legitim, dass dessen Erfüllung nur angestrebt, aber womöglich niemals vollumfänglich erreicht werden kann, womit es sich vom Geltungsanspruch allgemeinverbindlicher Normen (und Gesetze) unterscheidet. Aus Sicht der Wertorientierung von Unternehmen würde das Streben nach dem (moralisch) Guten so nur einen neben mehreren Wertebereichen in der Unternehmessteuerung zum Ausdruck bringen, wobei natürlich insbesondere auch ökonomische Werte legitimer Bezugspunkt der Orientierung wären. Eine verbindliche Handlungsanleitung lässt sich dabei kaum in einzelne Werte hineinschreiben, was ihre Funktion als Idealbilder und Leitgedanken jedoch nicht schmälert, sondern vielmehr ermöglicht.

\subsection{Zum Zusammenhang von Normen und Werten in der Unternehmens- steuerung}

Für die Auseinandersetzung mit Compliance und Integrity ergeben sich durch das im Ausgang der Kritik an Habermas deutlich gewordene Verweisungsverhältnis von Normen auf Werte weitere Einsichten. Insofern Normensysteme zur inhaltlichen Füllung auf Werte verweisen, zeigt sich, dass das verbreitete Verständnis von Compliance als Norm- oder Regelbefolgungsansatz und Integrity als Werteorientierungsansatz für eine analytisch fundierte Differenzierung zu kurz greift. Bereits die gängigen Gesetze, die im Bereich Compliance relevant sind, wie unter anderem Anti-Korruptions- oder Wettbewerbsgesetze, zielen darauf ab, eine Verwirklichung moralischer und außermoralischer

13 Diese Schwierigkeit ergibt sich nur vor dem Hintergrund einer Begriffsverwendung, wie wir sie etwa bei Habermas finden. Alternative Konzepte von „Wert“ können freilich auch von der Kategorizität von Werten ausgehen (vgl. Beschorner 2005: 47). 
Werte zu ermöglichen. Ebenso ist die Berücksichtigung von Regeln und Normen selbst Ausdruck einer bestimmten Haltung (vgl. Horster 2007: 210f.). Dementsprechend können auch bei einer Compliance-Orientierung bestimmte Wertvorstellungen identifiziert werden. ${ }^{14}$ Beispielsweise kann sich in der Rechtstreue eines Unternehmens tatsächlich eine moralische Wertehaltung widerspiegeln, insofern natürlich Steuerabgaben, Vertragstreue usf. selbst auch eine bewusste gesellschaftliche Verantwortung zum Ausdruck bringen können - wenn auch eine im Sinne des vorgestellten Verständnisses von Werten als Idealen und Leitbildern verkürzte. Ferner kann das Streben nach Compliance von ökonomischen oder anderweitig außermoralischen Werten motiviert sein, wenn es als ein Erfolgsfaktor erkannt wird. Schließlich basieren interne Compliance-Regeln oft auf konkreten ökonomischen Wertvorstellungen. So kann die Prävention von korruptem Verhalten durch die Beschäftigten deshalb für ein Unternehmen wichtig sein, weil es für sich definiert hat, dass es sein Geschäft auf der Basis von (impliziten) Werten wie Innovationsfähigkeit, Qualität oder durch Überzeugung seiner Kunden machen möchte und nicht aufgrund von fragwürdigen Zuwendungen, die das Geschäftsmodell möglicherweise auf Dauer untergraben würden.

Die Erkenntnis des Zusammenhangs von Normen und Werten macht damit die Unterscheidung zwischen Compliance und Integrity jedoch keineswegs hinfällig. Vielmehr kann sie helfen, die konzeptionelle Differenz beider Managementstrategien und damit deren jeweilige Rollen in der Unternehmenssteuerung klarer zu sehen. Auch wenn es zutrifft, dass Werte realisiert werden wollen und Normen hierzu Anleitungen darstellen, zeigt sich weiterhin eine relevante Akzentverschiebung zwischen Normen und Werten, die wir in die entsprechenden unternehmensethischen Strategien hinein spiegeln können. Normen teilen Handlungen als geboten oder nicht-geboten ein, wobei nicht-gebotene Handlungen entweder verboten oder erlaubt sein können. Werte, so wurde deutlich, können in ganz verschiedenen Graden realisiert werden. Die häufig im Zusammenhang mit Werten aber auch mit Integrity-Ansätzen betonte Rolle von Idealen oder Leitbildern deutet bereits an, dass Werte damit eine regulatorische Funktion haben und verglichen mit Normen nicht einmal darauf angelegt sein müssen, in vollumfänglichem $\mathrm{Maß}$ realisierbar zu sein bzw. Handlungen direkt anzuleiten. Damit eignen sie sich, um mittels des reichen Vokabulars an Wertbegriffen die Vision und Mission eines Unternehmens zu beschreiben und die Unternehmenskultur und -identität zu prägen. Wird diese Wertehaltung somit auch von den Unternehmensmitgliedern eingenommen, wirkt sich dies positiv auf das gewünschte Verhalten aus - auch wenn dieses ansonsten vornehmlich durch Regeln angeleitet ist. Für das Streben nach einer moralisch verantwortungsbewussten Unternehmensführung ist die Ausrichtung an Werten dagegen unabdingbar, da erst in ihnen das Gute, das realisiert werden soll, zum Ausdruck kommt.

14 Grundsätzlich könnte sich der Compliance-Begriff auch auf die Einhaltung von Werten beziehen, da er eine beliebige inhaltliche Füllung zulässt. In der Praxis ist jedoch ein Verständnis geläufig, das den Schwerpunkt eher auf gesetzliche Regeln, Standards und interne Richtlinien setzt und sich damit auf Regelungen bezieht, die konkrete Ansprüche an die Handlungen der betroffenen Akteure formulieren. Es geht dabei weniger um Vorstellungen des Guten, wie sie u.a. in Werten zum Ausdruck kommen, sondern mehr um klare Handlungsweisen (Gebote und Verbote), auf die man sich in einer Gesellschaft, einem Unternehmen o.ä. verständigt hat. 
Der festgestellte Unterschied zwischen den zwei Strategien lässt sich auch anhand normativer und evaluativer Sollens-Aussagen aufzeigen, die eingangs mit Normen und Werten in Verbindung gebracht wurden. Während Normen beschreiben, dass etwas getan werden soll, drücken Werte aus, dass etwas der Fall sein soll. Markant ist hier, dass das in der Ethik bekannte Diktum von „Sollen impliziert Können“, nach dem eine sinnvolle moralische Forderung prinzipiell erfüllbar sein muss, nur für den ersten Fall, nämlich für Normenurteile gilt, sich jedoch nicht auf Werturteile beziehen muss. Die Forderung zu stellen, dass es allen Menschen gut gehen soll, kann gerade durch keinen konkreten Akteur realisiert werden, da niemand hierzu faktisch die Handlungsmöglichkeiten hat. In gleicher Weise wäre es sinnlos mittels eines Compliance-Ansatzes im Unternehmen zu fordern, dass alle Beschäftigten stets gesund sein sollen. Dagegen wäre eine entsprechende Orientierung des Unternehmens am Wert der Gesundheit keineswegs eine sinnlose Forderung, die im Rahmen eines Integrity-Ansatzes Eingang in die Unternehmensführung finden kann. Bei einer solchen Forderung handelte es sich dann aber vielmehr um ein Leitbild oder einen Zustand, für dessen konkrete Realisierung der entsprechende Wert schließlich auch in verschiedene Regeln (Arbeitsschutz, Amtsarzt etc.) gegossen werden muss. Hier kann wieder die Compliance-Strategie zum Zug kommen. So lässt sich im Anschluss an Talaulicar formulieren: „soweit es nur möglich (...) ist, dass Werte als Auffassungen des Wünschenswerten Handlungen anleiten, müssen sie an Normen gebunden werden, um unzweideutig präskriptive Kraft zu erlangen“ (Talaulicar 2006: 348). Im Prozess dieser Anbindung, so lässt sich feststellen, kann aus Leitbildern eine konkrete Handlungsanleitung werden. In dieser Hinsicht können nun Werte- und Normenorientierung zusammen wirksam werden.

\section{Fazit: Das Ergänzungsverhältnis von Compliance und Integrity anhand der Normen- und Werteunterscheidung}

Die über das Verhältnis von Normen und Werten gewonnenen Einsichten können sowohl die Attraktivität von Integrity-Ansätzen klären, als auch deren Grenzen aufzeigen und somit die Kombination mit Compliance-Ansätzen plausibilisieren. Gerade weil wir es bei Werten zum Teil mit abstrakteren evaluativen Urteilen zu tun haben, eignen sie sich wie dargestellt als Leitvorstellungen, die den grundlegenden Rahmen einer Strategie der Unternehmensverantwortung bilden und inspirierend wirken. Wenn Paine gegen die von ihr als legalistisch und instrumentalistisch kritisierten Compliance-Ansätze hervorhebt, „law does not generally seek to inspire human excellence“ (Paine 1994: 111), verweist sie auf diesen Vorzug von Integrity-Ansätzen, sinnstiftend und richtungsweisend zu sein und entspricht damit in ihrer Argumentation auch der aufgezeigten Rolle von Werten in der Ethik. In ähnlicher Weise meinen auch Rasche und Esser (2007: 111) „Compliance cannot achieve inspiration because the approach focuses on prescriptions". Die Einführung von Werten in die Compliance-Diskussion verweist somit auf die Notwendigkeit von Leitbildern und Wertehaltungen in der Unternehmensführung genauso wie diese zur Motivation jedes menschlichen Handelns entscheidend sind. Um dies in der Konsequenz an einem Beispiel zu illustrieren, führen die zwei Ansätze bezüglich der Frage der Gesundheit der Beschäftigten beispielsweise zu unterschiedlichen Umsetzungsmaßnahmen, die beide ihre Berechtigung haben. In dem einen Fall liegt der Fokus auf der Einhaltung von statischen Normen und Regeln etwa des Arbeitsschutzes, 
im anderen Fall auf einem reflexiven Verhältnis zum Wert der Gesundheit, dessen Realisierung von dem spezifischen Verständnis und der Gewichtung dieses Werts innerhalb des Unternehmens geprägt ist und je nach Unternehmenskultur zu kreativen Ansätzen der Gesundheitsförderung führen kann. Hier zeigt sich, wie werte- und normenorientierte Ansätze in der Wirtschaftsethik unterschiedliche Rollen bei einer verantwortungsbewussten Unternehmenssteuerung spielen können.

Durch die Bewusstwerdung der im Vergleich zu Normen unverbindlicheren Geltungsansprüche von Werten wird aber auch deutlich, warum ein Integrity-Ansatz überladen würde, wenn er als alleiniges Instrument verantwortlicher Unternehmenssteuerung verstanden wird. Eine starke Fokussierung auf Werte, wie im Rahmen von Integrity, erlaubt nämlich schwerlich die Durchsetzung einer Null-Toleranz-Policy (vgl. Rasche/Esser 2007: 109), wie sie für nicht wenige Fragen des unternehmerischen Handelns gefordert wird, beispielsweise im Bereich des Exports (Gefahrgut, Embargos etc.). Hier wird gerade nach der Binarität und damit nach der Verbindlichkeit und Eindeutigkeit verlangt, die gemeinhin mit Normen und Regeln einhergeht und durch ein Compliance-System etabliert und überwacht werden kann.

Anknüpfend an das Merkmal von Werten, dass sie zwar durch ihre Orientierungsfunktion Verlässlichkeit schaffen, allerdings eben keine unbedingte Verbindlichkeit und Handlungsanweisung erzeugen, ist in der Rezeption des Integrity-Ansatzes zudem eine gewisse Spannung zu erkennen. So wird einerseits die identitätsprägende Natur von Unternehmenswerten und entsprechender Integrity-Strategien hervorgehoben. Erst durch diese würde schließlich der Raum geschaffen, um über moralische Anforderungen zu reflektieren und sie anhand der eigenen Werteorientierung zu adressieren (vgl. Wieland 2014: 32). Andererseits wird mit Integrity-Ansätzen zugleich der Anspruch verbunden, die mangelnde Wirksamkeit von Compliance-Strategien zu korrigieren und zu einer direkten Verhaltensänderung dort zu führen, wo Compliance Management versagt (vgl. u.a. Verhezen 2008). Damit wird der Integrity-Ansatz aber mit einer Forderung verbunden, die sich zumindest mit einem analytischen Verständnis von Werten als Orientierungsstandards nicht unmittelbar umsetzen lässt.

Bezüglich des Anliegens dieser Untersuchung, das Ergänzungsverhältnis der zwei behandelten Unternehmensstrategien zu beleuchten, erwies sich der Abgleich von Compliance und Integrity mit den ethischen Grundbegriffen von Normen und Werten als fruchtbar. Über den Weg der Diskursethik, mit ihrem rigiden Scheiden dieser beiden Konzepte, und der anschließenden Einsicht in die Problematik dieses Unterfangens, konnten wir Klarheit nicht nur über die Bedeutung von Werten für normenorientiertes Handeln gewinnen, sondern auch bezüglich der Frage, welche Rolle Werten in den verhandelten Ansätzen der Unternehmensethik zukommen kann. Wir haben dabei insbesondere die motivationale Bedeutung von Werten für verantwortliches Unternehmenshandeln sowohl bei einer an Compliance als auch einer an Integrity orientierten Unternehmenssteuerung aufgezeigt sowie ihre fundierende Rolle für entsprechende Handlungsregeln beleuchtet. Zugleich konnte durch diese Analyse ein Ergänzungsverhältnis von (regelgeleitetem) Compliance Management und (werteorientiertem) Integrity Management unterstrichen werden. Während sich viele Hoffnungen mit der Idee eines wertegeleiteten Managements verbinden, deutete sich aus der Untersuchung an, dass diese berechtigt sind, eine solche Strategie jedoch mit spezifischen Herausforderungen 
verbunden ist, die sich für einen Regelbefolgungsansatz so nicht stellen. Zugleich wurden aus analytischer Perspektive die Grenzen einer Normenorientierung ohne entsprechend fundierende Werte deutlich. Letztlich erwies sich damit die hier versuchte begriffliche Parallelisierung als ertragreich und stützt damit die Einsicht in die Notwendigkeit einer Compliance- und Integrity-Strategie als Instrument für eine verantwortungsbewusste Unternehmenssteuerung. ${ }^{15}$

\section{Literaturverzeichnis}

Beschorner, T. (2005): Integrität, Institution, Transformation, in: Zeitschrift für Wirtschafts- und Unternehmensethik, Jg. 6/Heft 1, 46-50.

Cox, D./La Caze, M./Levine, M. P. (2003): Integrity and the Fragile Self, Aldershot, Burlington (VT): Ashgate.

Fassio, D. (2013): How to Distinguish Norms from Values, in: Phenomenology and Mind, Heft 5, 148-158.

Gottschalk-Mazou₹, N. (2000): Diskursethik: Theorien, Entwicklungen, Perspektiven, Berlin: Akademie-Verlag.

Grüninger, S. (2011): Integrity Management, in: Hernsteiner, Heft 2, 9-10.

Grüninger, S. (2014): Werteorientiertes Compliance-Management-System, in: Wieland, J./Steinmeyer, R./Grüninger, S. (Hrsg.): Handbuch Compliance-Management: Konzeptionelle Grundlagen, praktische Erfolgsfaktoren, globale Herausforderungen, 2. Aufl., Berlin: Erich Schmidt Verlag, 41-70.

Habermas, J. (1983): Moralbewußtsein und kommunikatives Handeln, Frankfurt a.M.: Suhrkamp.

Habermas, J. (1991): Erläuterungen zur Diskursethik, Frankfurt a.M.: Suhrkamp.

Habermas, J. (1992): Faktizität und Geltung: Beiträge zur Diskurstheorie des Rechts und des demokratischen Rechtsstaates, Frankfurt a.M.: Suhrkamp.

Habermas, J. (1996): Die Einbeziehung des Anderen: Studien zur politischen Theorie, Frankfurt a.M.: Suhrkamp.

Habermas, J. (2000): Werte und Normen: Ein Kommentar zu Hilary Putnams kantischem Pragmatismus, in: Deutsche Zeitschrift für Philosophie, Jg. 48/Heft 4, 547-564.

Habermas, J. (2002): Die Zukunft der menschlichen Natur, Frankfurt a.M.: Suhrkamp.

Halfon, M. S. (1989): Integrity. A Philosophical Inquiry, Philadelphia: Temple University Press.

Höffe, O. (2002): Eintrag „Wert“, in: ders: Lexikon der Ethik, 6. Aufl., München: Beck, 290-291.

Horster, D. (2007): Das Verhältnis von Normen und Werten, in: Härle, W./Vogel, B. (Hrsg.): „Vom

Rechte, das mit uns geboren ist“. Aktuelle Probleme des Naturrechts, Freiburg: Herder, 202-215.

Joas, H. (1997): Die Entstehung der Werte, Frankfurt a.M.: Suhrkamp.

König, M. (2001): Das Drei-Schritt-Modell der Unternehmensethik am Beispiel der Diskursethik, in: Zeitschrift für Wirtschafts- und Unternehmensethik, Jg. 2/Heft 2, 155-180.

Krijnen, C. (2002): Wert, in: Düwell, M./Hubenthal, C./Werner, M.H. (Hrsg.): Handbuch Ethik, Stuttgart, Weimar: Metzler, 527-533.

15 Für hilfreiche Kommentare danken wir Prof. Dr. Stephan Grüninger, Christopher van Dillen sowie zwei anonymen Gutachtern der zfwu. 
Lumer, C. (1997): Habermas' Diskursethik, in. Zeitschrift für philosophische Forschung, Jg. 51/Heft 1, 42-64.

McFall, L. (1987): Integrity, in: Ethics, Vol. 98/No. 1, 5-20.

Paine, L. S. (1994): Managing for Organizational Integrity, in: Harvard Business Review, Vol. 72/No. 2, 106-117.

Putnam, H. (2002): Values and Norms, in: ders: The Collapse of the Fact/Value Dichotomy and Other Essays, Cambridge (MA)/London: Harvard University Press, 111-134.

Rasche, A./Esser, D. E. (2007): Managing for Compliance and Integrity in Practice, in: Carter, C. et al. (Eds.): Business Ethics as Practice: Representation, Reflexivity and Performance, Cheltenham, Northampton: Edward Elgar, 107-127.

Reese-Schäfer, W. (1997): Grenzgötter der Moral. Der neue europäisch-amerikanische Diskurs zur politischen Ethik, Frankfurt a.M.: Suhrkamp.

Steinmann, H./Olbrich, T. (1998): Business Ethics in U.S.-Corporations. Results from an Interview Series, in: Ulrich, P./Wieland J. (Hrsg.): Unternehmensethik in der Praxis: Impulse aus den USA, Deutschland und der Schweiz, Bern/Stuttgart/Wien: Haupt, 63-89.

Talaulicar, T. (2006): Unternehmenskodizes: Typen und Normierungsstrategien zur Implementierung einer Unternehmensethik, Wiesbaden: Deutscher Universitäts-Verlag.

Tappolet, C. (2013): Evaluative vs. Deontic Concepts, in: LaFollette, H. (Ed.): The International Encyclopedia of Ethics, New York: Blackwell, 1791-1799.

Tappolet, C. (2015): Value and Emotions, in: Hirose, I./Olson, J. (Eds.): The Oxford Handbook of Value Theory, Oxford: Oxford University Press, 80-95.

Thielemann, U. (2005): Compliance und Integrity - Zwei Seiten ethisch integrierter Unternehmenssteuerung. Lektionen aus dem Compliance Management einer Großbank, in: Zeitschrift für Wirtschafts- und Unternehmensethik, Jg. 6/Heft. 1, 31-45.

Ulrich, P. (2004a): Wirtschaftsethische Graswurzelreflexion statt angewandte Diskursethik: Persönliche Sicht einiger Differenzen zwischen St. Galler und Berliner Position, in: Bausch, T./Böhler, D./Rusche, T. (Hrsg.): Wirtschaft und Ethik: Strategien contra Moral?, Münster: Lit, 21-42.

Ulrich, P. (2004b): Normative Orientierungsprozesse, in: Dubs, R./Euler, D./Rüegg-Stürm, J./Wyss, C. E. (Hrsg.): Einführung in die Managementlehre, Bd. 2, Bern/Stuttgart/Wien: Haupt, 23-37.

Ulrich, P. (2008): Integrative Wirtschaftsethik. Grundlagen einer lebensdienlichen Ökonomie, 4. Aufl., Bern/Stuttgart/Wien: Haupt.

Verbezen, P. (2008): The (Ir)relevance of Integrity in Organizations, in: Public Integrity, Vol. 10/No. 2, 135-152.

Wieland, J. (2008): Die Kunst der Compliance, in: Löhr, A./Burkatzki, E. (Hrsg.): Wirtschaftskriminalität und Ethik, DNWE Schriftenreihe, Bd. 16, Mering: Rainer Hampp Verlag, 155-169.

Wieland, J. (2014): Compliance Management als Corporate Governance - konzeptionelle Grundlagen und Erfolgsfaktoren, in: Wieland, J./Steinmeyer, R./Grüninger, S. (Hrsg.): Handbuch Compliance-Management: Konzeptionelle Grundlagen, praktische Erfolgsfaktoren, globale Herausforderungen, 2. Aufl., Berlin: Erich Schmidt Verlag, 15-40.

Wieland, J./Steinmeyer, R./ Grüninger, S. (Hrsg.) (2014): Handbuch Compliance-Management: Konzeptionelle Grundlagen, praktische Erfolgsfaktoren, globale Herausforderungen, 2. Aufl., Berlin: Erich Schmidt Verlag. 